\title{
Desafíos para la institucionalización de la perspectiva de género en instituciones de educación superior en México. Una mirada a los contextos organizacionales.
}

Challenges for the gender institutionalization in institutions of higher education in Mexico. A view to the organizational contexts.

Dra. Daniela Cerva Cerna*

\section{Resumen}

El artículo intenta analizar el devenir de los estudios de la mujer y el género en el marco de los desafíos que enfrentan las instituciones educativas en su participación en la producción de conocimientos sobre las mujeres y las relaciones de género, poniendo de relieve la importancia de generar acciones institucionales sobre las propias dinámicas que reproducen las relaciones de poder dentro de los espacios educativos. El análisis incluye los resultados de investigaciones cualitativas con mujeres académicas e integrantes de las unidades de género en algunas universidades mexicanas.

Palabras Claves: Universidades - Institucionalización del género - cultura organizacional - brechas de género - mujeres académicas

\begin{abstract}
This article seeks to examine what happens to women's studies and gender with respect to the challenges facing educational institutions in their participation in the production of knowledge on women and gender relations to highlight the importance of generating institutional actions on the own dynamics that reproduce power relations within the educational spaces. The analysis includes the results of qualitative research with academic women and members of gender units in some Mexican universities.
\end{abstract}

Key words: Universities - gender institutionalization - organizational culture - gender gap-academic women

Fecha de recepción: 17 de febrero 2017

Fecha de aprobación: 17 de enero 2017

\footnotetext{
* Socióloga por la Universidad de Chile, Maestra en Ciencias Sociales por la FLACSO México, Doctora en Ciencias Políticas y Sociales por la Facultad de Ciencias Políticas y Sociales de la UNAM, Egresada Posdoctorado CRIM-UNAM. Actualmente es Profesora Investigadora Tiempo Completo en la Facultad de Derecho y Ciencias Sociales de la UAEM México. Líneas de Investigación: Políticas públicas, gobernanza, y análisis organizacional. Representación y participación política. Estudios feministas y de Género.
} 


\section{Introducción}

Abrir las universidades y centros de educación superior a los estudios feministas y de género representa una gran oportunidad, con muchos retos y desafíos, incluyendo tanto el desarrollo de un conocimiento científico que analice y conozca las consecuencias que tiene en todos los ámbitos de la vida la forma en que se construyen las relaciones de género, así como en el despliegue de una estructura institucional que le dé sustento y permita su consolidación y legitimidad como campo de estudio y como un aspecto propio del funcionamiento interno de los centros de educación superior .

En este proceso emerge un importante cuestionamiento a las propias dinámicas culturales y políticas presentes en las universidades, aspecto fundamental a la hora de analizar cómo se incorpora la perspectiva de género como un valor que oriente las relaciones sociales que son parte de este espacio social.

Por lo anterior, la reflexión que propongo intenta analizar el devenir de los estudios de la mujer y el género en el marco de los desafíos que enfrentan las instituciones educativas en su participación en la producción de conocimientos sobre las mujeres y las relaciones de género, poniendo de relieve la importancia de generar conocimiento sobre las propias dinámicas que reproducen las relaciones de poder dentro de los espacios educativos y la necesidad de establecer acciones para este fin.

El trabajo se organiza en tres apartados. El primero expone, a grandes rasgos, la trayectoria de los estudios de género, junto con la demanda sostenida por institucionalizar espacios de incidencia dentro de las universidades; posteriormente se revisa, a partir de la experiencia mexicana, las tensiones institucionales y organizacionales que este objetivo conlleva, enfatizando la interpretación y experiencia que algunas académicas activistas hacen sobre los avances y límites que encierra la incorporación de mujeres y del enfoque de género en los centros de educación superior. Finalizamos con algunas propuestas que se ponen a discusión desde una revisión crítica del enfoque de la institucionalización en espacios generizados ${ }^{1}$.

\section{Los estudios de las mujeres y el género en las universidades}

Al revisar el devenir de los estudios e investigación feminista en México y América Latina, es posible sostener que son casi cuatro décadas en que la investigación sobre las relaciones de género se ha posicionado en el proceso de conocimiento e investigación

\footnotetext{
${ }^{1}$ Como he sostenido en otros escritos, las organizaciones no son neutrales, son espacios que surgen de ciertos contextos institucionales y en su base producen y reproducen relaciones de poder que tienen un efecto en la desigualdad de género. Los espacios generizados reproducen una determinada forma de masculinidad: la hegemónica, esto influye en la percepción y práctica sobre la implementación de acciones institucionales de género y su disposición al cambio se encuentra condicionada a la lógica de la cultura política y organizacional.
} 
académica, en la práctica y reflexión de organizaciones y movimientos sociales, en organismos internacionales y más recientemente en la esfera del Estado a través de las políticas públicas y la legislación nacional.

Este avance no ha sido una tarea fácil. Si bien hoy la perspectiva de género cuenta con amplia legitimidad y reconocimiento como una categoría explicativa de la forma en que se construye social y culturalmente la diferencia sexual, en sus inicios no estuvo exenta de críticas y cuestionamientos, incluso dentro del propio feminismo ${ }^{2}$.

Lo mismo puede decirse de los espacios productores de conocimiento, los que históricamente excluyeron e invisibilizaron la contribución de las mujeres a las distintas disciplinas científicas, ya sea negándoles el acceso a la formación profesional o limitando su participación en los espacios y fuentes de producción y legitimación de los saberes. La ciencia, como otras esferas de lo público-político-social, se ha enfrentado a la constante crítica y revisión que desde el feminismo se ha hecho de su práctica (Blázquez, et. al. 2010), con la intención de desmontar sus presupuestos patriarcales y su mirada androcéntrica, y a la vez contribuir con la formulación de nuevas preguntas y enfoques que cuestionan las teorías y enfoques explicativos dados por objetivos y universales.

La revisión crítica de la actividad científica se asienta dentro de un marco histórico que se vio fortalecido por las exigencias de los movimientos sociales y de mujeres, quienes denunciaron la invisibilidad de las mujeres como sujetos y objeto del conocimiento que se daba por universal y objetivo, y demandaron diagnosticar la posición y condición de las mujeres en distintos ámbitos de la vida. Militancia feminista y académica han ido de la mano, y se han visto fortalecidas por los encuentros regionales e internacionales para la promoción de los derechos de las mujeres, que desde mediados de los setenta han incidido en impulsar una agenda feminista de investigación y propuestas normativas y de políticas públicas.

En un inicio denominados estudios de la mujer, y posteriormente estudios de género, la investigación sobre las condiciones de discriminación hacia las mujeres se revistió de la experiencia y apoyo de académicas que pertenecían a su vez a organizaciones feministas. Su objetivo fue académico y político al querer democratizar los espacios productores de conocimiento que han excluido históricamente a las mujeres como sujetos y objetos de estudio científico.

En nuestro país, como en otros de América Latina (Belluci 1992 y León 2007) el impacto de los estudios de género se dejó sentir con mayor fuerza en el campo humanístico y de las ciencias sociales, específicamente en disciplinas como la antropología, historia, sociología, psicología, letras, comunicación.

\footnotetext{
${ }^{2}$ Ver por ejemplo la compilación de Silvia Tubert, 2011 Del sexo al género. Los equívocos de un concepto, Editorial Cátedra.
} 
En el caso mexicano, los programas de estudio, así como las unidades de género dentro de las universidades, se inician a mediados de los ochenta ${ }^{3}$, consolidándose a principios del siglo XXI en varios centros de estudio universitario, aspecto que coincide con la propia institucionalización de la perspectiva de género en el Estado.

A mi juicio este hecho ha contribuido a un mayor empuje y legitimidad del conocimiento que debe desarrollarse dentro de las instituciones de educación superior. El Estado se convierte en un actor que demanda investigación, diagnósticos sobre la situación de las mujeres y estrategias para generar políticas públicas acordes a las necesidades de esta población, pero por sobre todo, requiere de formación y habilitación profesional para las y los funcionarios vinculados con las políticas de género en el Estado. Así es como hoy en día en México encontramos una serie de programas de estudio, especialidades, seminarios, diplomados, cursos de posgrado, entre otros, sobre género y políticas públicas vinculados a temáticas específicas como violencia, participación política de las mujeres, etc.

Como sostiene Marcela Lagarde (1996), el análisis de género feminista, contiene de manera explícita una crítica a los aspectos nocivos, destructivos, opresivos y enajenantes que se producen por la organización social basada en la desigualdad, la injusticia y la jerarquización política de las personas basada en el género. Como categoría ha sido fundamental para dar cuenta de una realidad que se tenía por dada, naturalizando las desigualdades y desventajas de la construcción y significación social de género de las sociedades e identidades personales. En este sentido, el enfoque de género ha servido para analizar y comprender la condición y la situación de mujeres y hombres.

Claramente estamos en presencia de un impacto importante de la investigación feminista y de género que sale de los márgenes de la academia para convertirse en un enfoque que informa y da sustento a los procesos políticos de toma de decisión. Sin duda este aspecto ha sido un gran avance, como señala Belluci (1992), si en un momento el objetivo fue hacer visible lo que se mostraba invisible para la sociedad y con ello desmontar la pretendida naturalización de la división socio-sexual del trabajo, la exclusión en lo público y su sujeción en lo privado, así como cuestionar la retórica presuntamente universalista de la ideología patriarcal, hoy en día ese conocimiento ha salido de los centros de estudio feminista influyendo en esferas relacionadas con la toma de decisión pública.

\footnotetext{
${ }^{3}$ Mary Golsmith hace un recuento y señala que los primeros cursos se impartieron en la Escuela Nacional de Antropología e Historia y la Facultad de Ciencias Políticas de la UNAM. La formación de centros y programas académicos se da en los ochenta, el Centro de Estudios de la Mujer de la UNAM, el Programa Interdisciplinario de Estudios de la Mujer en El Colegio de México y el área de investigación Mujer, identidad y Poder, de la UAM Xochimilco. Golsmith, M, 1998.
} 
Sin embargo, en algunos casos se ha incorporado la mirada de género de manera acrítica, con un uso casi aséptico que invisibiliza las relaciones de poder que están en su base. Como sostiene Magdalena León (2007), en algunos sentidos la utilización del enfoque de género trajo aparejada consecuencias no esperadas. La escisión entre demandas feministas e investigación aplacó el contenido reivindicativo, neutralizando la génesis de la teoría feminista. Los requisitos de financiamiento de las universidades y del propio trabajo de investigación de las académicas explican en gran medida la tecnificación o burocratización del enfoque de género.

En efecto, las lógicas institucionales y organizativas de los espacios académicos restringen la consolidación de un pensamiento crítico que exige la propia mirada al espacio desde donde el conocimiento de género se produce.

Quizás este es el aspecto más complejo que es preciso revisar a la luz no sólo de la propia legitimidad que los estudios de género tienen dentro de los espacios de educación superior; las dinámicas de promoción y mérito exigidas en base a una supuesta neutralidad de género como una cualidad dentro de los criterios de evaluación, son una muestra de cómo se reproducen las brechas y sesgos de género en los ambientes académicos ${ }^{4}$.

Como bien señala Ana Guil (2004), las creencias estereotipadas sobre las capacidades de las mujeres se siguen reflejando en las políticas discriminatorias organizacionales implícitas, que admiten con facilidad a las mujeres en los puestos más bajos del escalafón, a la vez que dificultan de manera naturalizada el que puedan llegar a asumir los más altos cargos de responsabilidad. Ello las obliga, de forma solapada "a constantes renuncias personales y/o familiares si pretenden optar a dichas posiciones directiva, renuncias que jamás se llegan a plantear sus colegas varones" (2004:16).

Por su parte, Louise Morley $(1999,2010)$ advierte para el caso británico, que si bien la composición de género en las organizaciones de educación superior ha ido cambiando, aún persisten fuertes barreras estructurales y actitudinales en relación al papel que cumplen las mujeres como productores de conocimiento. Varias investigaciones dan cuenta que en general ellas postulan a becas de investigación en menor proporción que sus colegas varones y son menos elegidas en investigaciones financiadas, por tener nombramientos de baja categoría. A menudo se sitúan en la investigación como

\footnotetext{
${ }^{4}$ Para el caso de las académicas de la UNAM, la valoración diferencial que se ha establecido en torno a lo que se considera "más productivo" como criterios de evaluación las pone en desventaja frente a sus pares varones. Nos referimos a la docencia versus publicar y hacer investigación. Pese a lo que significa estar frente a grupo y el trabajo que implica la formación, la docencia es un aspecto percibido como desventajoso y poco valorado para el desarrollo profesional (Cerva, 2011). Publicar y liderar grupos de investigación son las variables necesarias para fortalecer los indicadores de ascenso y jerarquía académica.
} 
asistentes y no en lugar de directores de investigación. Incluso en Suecia, Wenneras y Wold (1997) encontraron que los criterios de elegibilidad de género dan cuenta que las mujeres debían ser dos veces y media más productivas que los hombres para obtener la misma calificación en las competencias científicas.

Estos hechos están influyendo en la percepción que las académicas jóvenes tienen sobre los costos y las prioridades en materia de desarrollo profesional. El ambiente de competencia, en extremo individualista y apegado a la productividad, es internalizado como el mandato principal por parte de las nuevas generaciones de académicas y académicos, lo que está influyendo en el caso de las mujeres, en posponer o limitar una posible aspiración a la maternidad, así como afectar su salud física y emocional dado el estrés generado por la necesidad de demostrar productividad (Cerva 2011).

\section{Transformaciones institucionales a favor de la igualdad de género en las Universidades}

En relación a la presencia del enfoque de género en el campo del saber universitario, es importante destacar que los avances en materia de investigación y producción de conocimientos sobre las relaciones de género se manifiesta no sólo en los cursos o programas desarrollados, cuentan también las revistas especializadas que se han consolidado en los último años ${ }^{5}$, las publicaciones científicas, el desarrollo de congresos nacionales e internacionales, las redes de trabajo, la producción de tesis de posgrado, etc.

Por lo anterior, considero que estamos en un momento clave en donde es preciso dar un paso más adelante del proceso de consolidar los estudios de género en las universidades hacia la necesidad de analizar a las universidades e instituciones de educación superior como espacios con género. En este sentido cobra relevancia preguntarse qué ha aportado el ingreso de los estudios de género a la transformación institucional en las universidades. Es decir, ¿̇e ha desarrollado un proceso paralelo de investigación de las propias condiciones de producción de conocimiento y del ámbito donde éste se despliega, más allá de lo que se produce?

Investigadoras como Buquet (2010, 2011, 2013), Palomar (2011), Vélez (2007, 2008), entre otras, han emprendido un trabajo sistemático de revisión de las condiciones, oportunidades y obstáculos que enfrentan las dependencias educativas en el proceso de incorporación de la perspectiva de género, no sólo en el ámbito educativo, sino que también político-organizacional.

${ }^{5}$ Por mencionar algunas: Revista La Ventana del Centro de Estudios de Género de la Universidad de Guadalajara; Revista GénEros, de la Universidad de Colima; Revista Interdisciplinaria de Estudios de Género de El Colegio de México; Revista Debate Feminista, desde 2016 parte del Centro de Estudios de Género de la Universidad Nacional Autónoma de México. 
De la revisión de los trabajos de estas investigadoras puedo concluir que a groso modo existen dos fenómenos que es preciso destacar:

1. Por una parte se ha legitimado la producción de conocimiento que se fundamenta en la perspectiva de género, pero su puesta en práctica no se da en el vacío, es parte de dinámicas organizacionales que responden a lógicas institucionales que se asientan en dinámicas desiguales de género y que se expresan en todos los ámbitos de las relaciones: laborales, estudiantiles, administrativas, normativas, etc., teniendo un impacto en la forma en que se legitima el conocimiento y los actores que lo promueven.

2. La idea de transversalidad ${ }^{6}$ del género, aun cuando pueda ser parte del léxico común, es una práctica que está lejos de manifestarse dentro de la IES. Es común encontrar grupos específicos de trabajo circunscritos a espacios o unidades de género que escasamente permean otras esferas de las IES, ya sea en términos de producción de conocimiento, como de trasformaciones político administrativas. Se repite, en este sentido, la ghetización de estos espacios y el sacrificio personal que está detrás de las políticas que se intentan promover.

Estos dos fenómenos implican una serie de situaciones cotidianas que se normalizan, pese a lo difundido de la noción de género como un enfoque crítico que intenta visibilizar y revertir situaciones de discriminación y desigualdad.

En términos concretos, que la perspectiva de género permee el trabajo cotidiano que se realiza al interior de las IES no ha contado con el mismo grado de avance que su inclusión como campo de estudio. Plantear la necesidad de mirar a la propia institución y a los sujetos genéricos que la conforman ha sido un trabajo mucho más complejo, porque requiere tomar una posición política que redunde en medidas para transformar la desigualdad estructural al interior de la institución.

Es decir, la ideología de género no sólo afecta en términos de estereotipos y roles a varones y mujeres de manera individual. Hay un entramado simbólico que forma parte de las propias estructuras sociales que definen y organizan espacios sociales en donde la interacción humana cobra vida. Es por ello que no basta solo con incluir mujeres en términos cuantitativos sino se trabaja con el aspecto simbólico del género.

\footnotetext{
${ }^{6}$ La transversalidad de la perspectiva de género es una estrategia que apunta al cambio estructuras de las instituciones en función de incorporar la igualdad de género como un principio rector de su quehacer. Es un trabajo que requiere voluntad política y compromiso de parte de los integrantes de la organización para producir cambios que vayan más allá de proyectos específicos. Hablamos que independiente de cual sea la misión de la institución, sus recursos, procesos, objetivos y productos consideren la situación específica de mujeres y hombres.
} 
Parto de dos premisas fundamentales. La primera es que las universidades e instituciones de educación superior (IES) son espacios privilegiados por la resonancia social para difundir y consolidar una mirada que cuestione la discriminación y desigualdad, y por tanto su misión en ámbitos de la educación y formación profesional puede favorecer de manera sustantiva a transformaciones sociales más profundas a favor de la igualdad. En segundo lugar, y relacionado con la primea premisa, las IES y universidades, como instituciones, no lograrán producir cambios sustantivos que trasciendan hacia lo social si no desarrollan un proceso sostenido de transformación de sus propias prácticas y funcionamiento organizativo con un compromisos a favor del género. El conocimiento que se construye a partir de la incorporación de la categoría de género, debe ir de la mano de procesos de transformación institucional de los espacios desde donde ese conocimiento es producido, difundido y sobre todo, legitimado (Cerva, 2008)

Cobra sentido traer a colación el análisis feminista de Diana Maffía $(2007,2010)$, en el sentido que la ciencia, y yo agregaría el espacio donde ésta se produce, debe considerarse en su doble aspecto como proceso y producto, y que en ambos casos es sexista. Como producto nos señala que hay un sexismo que ha sido develado por muchas epistemólogas y críticas feministas mediante la revisión y deconstrucción de teorías científicas; y hay otro sexismo que se vive como proceso en la composición y exigencias de pertenencia y méritos en las comunidades científicas para las propias mujeres en su adscripción profesional.

Como producto, hemos visto que gracias al esfuerzo feminista se ha desarrollado un saber que incorpora el conocimiento sobre las condiciones de vida de las mujeres en ámbitos como la historia, la economía, la política, la medicina, etc. Como procesos el aporte de la sociología del conocimiento fue sumamente útil al abrir un debate sobre la supuesta neutralidad del conocimiento y poner en escena los intereses ideológicos, de clase, raza y género de quienes intentan mantener sus privilegios es este campo.

Durante las últimas cuatro décadas el feminismo ha revisado la historia de exclusión y segregación de las mujeres en las instituciones de la ciencia, especialmente enfocando el limitado acceso de las mujeres a los medios de producción científica y su status dentro de las profesiones. Aquí es donde surge la investigación sobre mujeres y equidad de género en las universidades, destacando el interés por conocer la forma en que las mujeres participan de la producción de conocimiento y de las características sexistas y androcéntricas que este entorno supone.

La mayoría de estas investigaciones coinciden en subrayar que la segregación disciplinar, es decir, que muy pocas mujeres ingresen a carreras como ingeniería o matemáticas, responde a procesos de socialización diferenciales, junto con esquemas de discriminación dentro de las universidades. Así mismo, que mujeres que se dedican a la vida académica no cuenten con los mismo niveles de reconocimiento y prestigio que sus 
pares varones, da cuenta de la cargas diferenciales que pesan sobre ellas al ser las únicas responsables de mantener y conciliar vida familiar y trabajo (Buquet, 2008, 2013, Cerva, 2011). Su desempeño, es este sentido, en muchos casos está condicionado por la difícil que resulta la disponibilidad y dedicación exclusiva a sus carreras profesionales.

Así mismo, aquellas mujeres que se presentan como excepcionales por sus contribuciones al desarrollo científico no escapan del encuadre de valoración masculino en la definición del mérito y la excelencia. Como sostiene Maffia (2007) el problema del sexismo en ciencia no ha sido sólo, ni principalmente, el de establecer diferencias entre hombres y mujeres, sino fundamentalmente la jerarquización de esas diferencias, siempre desventajosas para el mismo género y que dio respaldo a una desigual repartición de los roles sociales.

Así, son estas dos esferas en donde la incorporación de la perspectiva de género cumple una doble función: como construcción de conocimiento y como transformación política de las instituciones. Sin embargo considero que a este diagnóstico es preciso añadir otro ámbito de práctica sexista presente en el desarrollo científico: el aspecto organizativo sobre el cual ésta se sostiene. Es decir, más allá de la investigación sobre género y de las condiciones genéricas de quienes lo producen, hay una dinámica espacial-organizativa que incluye actores (no sólo académicos) procesos (no sólo investigación o docencia) y estructuras (no sólo formales).

Por tanto, más allá de la producción de conocimiento sobre el género y de la participación de mujeres en la ciencia, hoy en día las universidades se ven exigidas a generar instancias que promuevan una cultura de igualdad. En el caso de México, este proceso ha ido de la mano de la propia dinámica del Estado en torno a las políticas de género, favoreciendo con recursos su promoción dentro de las Universidades.

Resultados de entrevistas en profundidad realizadas a las representantes de las Unidades/oficinas/Programa de género de algunas Universidades en México ${ }^{7}$ vienen a corroborar los hallazgos de investigaciones sobre institucionalización de la perspectiva de género en el Estado ${ }^{8}$. Es decir, aun cuando los objetivos institucionales sean distintos, hay importantes similitudes.

\footnotetext{
7 Se incluye: Universidad de Sonora, Universidad Autónoma Benito Juárez de Oaxaca, Universidad de Guadalajara, Universidad Autónoma Metropolitana, Universidad Autónoma de México, Universidad Autónoma del Estado de Morelos, Universidad Autónoma del Estado de México.

${ }^{8}$ Entre otros ver: Cerva, 2016 y 2017.
} 
A grandes rasgos, estas experiencias demuestran que la gestión del área de género dentro de las universidades ha significado ${ }^{9}$ :

1. Creación de redes de trabajo entre las unidades de género de las distintas universidades en el país que impulsan proyectos y actividades conjuntas, no obstante adolecen de continuidad y apoyo institucional. El compromiso y entrega de sus integrantes refuerza en muchos casos la sobre saturación de trabajo que las especialistas en género tienen dentro de las organizaciones públicas.

2. Apoyo en recursos de parte del gobierno federal (proyectos de investigación sobre diagnósticos institucionales, cursos, capacitación, etc) a través del Instituto Nacional de las Mujeres para formar las unidades dentro de las Universidades, los que posteriormente no se sostienen por las propias universidades ${ }^{10}$.

3. Cambios en la administración y autoridades universitarias que afectan en la falta de continuidad del personal, así como en la orientación de las acciones. Incluso en algunos casos la unidad llega a desaparecer si no hay voluntad y compromiso institucional ${ }^{11}$.

4. La retórica de la igualdad se instala como un compromiso que se deja ver principalmente para ciertas fechas emblemáticas: 8 de marzo y 25 de noviembre.

5. La perspectiva de género es percibida como amenaza para las propias mujeres académicas al contraponer el mérito exigido versus la supuesta discrecionalidad de las acciones afirmativas.

6. Desgaste y frustración de parte de las integrantes de las unidades de género, que incluso genera competencia interna por la "propiedad del tema", debido a la falta de espacios y recursos existentes para desarrollar investigación y docencia.

7. Escasa incidencia y participación en instancias y comités académicos donde se negocian y definen situaciones relativas al trabajo y vida universitaria.

\footnotetext{
${ }^{9}$ La creación de oficinas o instancias abocadas a la generación de acciones dirigidas a superar las desigualdades entre los sexos forma parte de una amplia gama acciones establecidas para incorporar la perspectiva de género en las instituciones.

${ }^{10}$ Como lo señala una de las entrevistadas "La administración de las universidades no planean presupuestalmente más que para operaciones administrativasy las unidades académicas priorizan sus recursos, y el género no es una prioridad, la formación de personal con perspectiva de género y los recursos que ofrece la Secretaría de Educación Pública son siempre insuficientes. Falta recursos etiquetados especificamente para estas actividades"(Universidad Autónoma Benito Juárez de Oaxaca Junio de 2016)

11 "A grandes rasgos, resistencia por parte del personal a nivel dirección, no existe un interés en la temática ya que desde su perspectiva no existen desigualdades dentro del ámbito universitario... Desconocimiento del tema". (Universidad de Sonora, Marzo 2016)
} 
8. Los espacios que forman parte del entorno de las organizaciones universitarias mantienen una fuerte carga simbólica masculina ${ }^{12}$.

Las universidades, como espacio educativo y de formación son representadas como un ámbito de saber, de inclusión e igualdad, sin embargo los resultados de estas indagaciones dan cuenta de situaciones que no son muy halagadoras y que ponen en escena una realidad incómoda. A la luz de las transformaciones en varios ámbitos institucionales para promover una participación equitativa e incluso paritaria de mujeres y hombres, la discriminación que se vive en las universidades se torna un ámbito que debe ser analizado.

En este sentido, investigadores feministas concuerdan en señalar que la representación de las universidades bajo un modelo de funcionamiento supuestamente igualitario ha sido un factor que ha naturalizado las prácticas discriminatorias y ha restringido el despliegue de una mirada crítica que propugna por generar medidas para su eliminación. Es preciso entender por tanto que el contexto patriarcal también se manifiesta en los espacios en donde el conocimiento se produce y que éste también está determinado por estructuras y dinámicas organizacionales que refuerzan una cultura organizacional que reproduce estereotipos y patrones sexistas.

Un ejemplo de ello es lo que nos señala Magdalena León (2007) al reconocer que si bien hoy en día los estudios de la mujer y los programas de género están presentes en la mayoría de las universidades de América Latina, estos proyectos son marginales en las prioridades científicas y financieras. Aparecen como esferas separadas de la corriente principal de la producción y son evaluadas como elementos que es preciso añadir a las universidades. Otro ejemplo lo constituyen los datos sobre los estímulos y fomento a la producción académica, que en rigor beneficia más a los varones que a las mujeres, así como las oportunidades de ascenso también son diferenciales, lo que se expresa en que los cargos de decisión estén ocupados por un mayor número de hombres.

Es interesante contrastar los fenómenos de discriminación en este ámbito con la falta de reconocimiento y aceptación de parte de la comunidad universitaria de las condiciones de género que actúan como variables explicativas de las condiciones de género en las universidades. Ejemplo de ello son las situaciones de violencia y acoso estudiantil y laboral que se encuentran sumamente normalizadas por estudiantes, académicos y personal administrativo.

Otro ejemplo muy interesante al respecto es el caso de las mujeres académicas e investigadoras que, cuando se les interroga sobre situaciones discriminación laboral y

12 "Y esto se ve en los temas de acoso y hostigamiento, son siempre colegas varones los implicados y entre ellos se tapan las cosas, prevalecen esos códigos de compartir ese poder que les da ser autoridad y docente, todos sabemos que andan con las alumnas o que las presionan en ese sentido" (Universidad Autónoma del Estado de Morelos, Noviembre 2016) 
profesional, tienden a no reconocerla y en algunos casos hasta negarla, adjudicando aspectos personales como motivo del por qué algunas mujeres no ascienden en su carrera académica (Cerva, 2011)

En este punto quisiera hacer un símil con la situación de la subrepresentación de las mujeres en la política ${ }^{13}$, de lo que sucede con las mujeres en la vida académica. El espacio público del poder político, así como el de las instituciones educativas se ha conformado bajo un modelo masculino que desde sus inicios se esforzó en excluir el ingreso de las mujeres. Así como ellas lucharon (y lo siguen haciendo) para ser aceptadas en el terreno político, en los centros educativos también han demandado mayor inclusión y reconocimiento de las distintas situaciones que por su género pueden ser constitutivas de desigualdad profesional. El gran problema es que tanto en la política como en la academia subsiste una visión de supuesta igualdad de derechos basada en la lógica de que el derecho ya existe una vez que se enuncia (mujeres pueden estudiar/votar) y en consecuencia su presencia en política como en la academia estaría ligada exclusivamente al principio del mérito, la capacidad y esfuerzo personal, aspectos que en su conjunto invisibilizan toda una estructura genérica que reproduce y alienta la exclusión de las mujeres del espacio público.

En efecto, tanto en el mundo político como en el educativo se esgrimen argumentos ligados con el mérito o las capacidades de quienes pueden ingresar a estas esferas o que están en posiciones superiores. Como señalan Sara Rietti y Diana Maffia (2002), la pregunta que es necesario hacerse en estos casos, es cómo se han construido dichos criterios del "mérito" como medidas para valorar el ingreso o no de las mujeres. Cuando las mujeres no cumplen con los méritos requeridos, el problema es de ellas, no de lo que se supone refleja esa norma de evaluación. Es decir, si las mujeres desde que obtuvieron el voto no están en política es por su incapacidad para adecuarse y ser parte de un esquema que aparenta ser neutral e igualitario. La experiencia en este ámbito nos ha demostrado que la supuesta ciudadanía universal se construyó sobre la base de la exclusión de las mujeres, y que han sida necesarias una serie de medidas que de manera intencional promuevan el ingreso de las mujeres en la vida política. Es por ello que un análisis del mérito versus las oportunidades permite generar una reflexión mucho más profunda y contextual al respecto.

Cuando hablamos del mérito, que es central en el sistema educativo como en el político, es decir, deben ingresar los mejores, olvidamos que su contenido se sustenta en las valoraciones culturales que afectan tanto a varones como a mujeres. En el discurso, entonces, el acceso estaría sustentado en la voluntad, habilidad y esfuerzo personal y no se incluye la mirada sobre la forma en que funcionan estos espacios como estructuras institucionales. Es decir, hay una forma de hacer política, así como de hacer ciencia que

${ }^{13}$ También se cuentan las mujeres en los ámbitos empresariales, financieros, religiosos, entre otros. 
se rige por ciertas prácticas y valores sobre los cuales no han sido socializadas las mujeres: el individualismo, la competencia, las jerarquías, etc. (Riatti y Maffia, 2002)

Es decir, ciencia y política son ámbitos institucionalizados que manifiestan órdenes de género que contribuyen a reproducir una estructura de suyo generizadas. $Y$ esta estructura privilegia lo masculino como condición de funcionamiento y su aceptación. Aunque con conflictos al comienzo, las mujeres van incorporando estos valores predominantes, por ello no es de extrañar que muchas veces a las mujeres en política se las achaque de masculinizarse y ser incluso más autoritarias que los propios varones.

\section{Institucionalizar la perspectiva de género (IPG) en los IES y universidades}

Al reconocer el papel de las instituciones en la reproducción y construcción de la desigualdad de género, también se asume que pueden tener un papel decisivo en contrarrestar estas situaciones a partir de iniciativas y políticas específicas, por ejemplo, reformas normativas, creación de mecanismos o unidades de género, generación de políticas institucionales entre otras medidas. En función de esto quiero hacer algunas precisiones conceptuales, todas necesarias para entender por qué es necesario introducir el género en la Universidades, desde qué niveles y con qué criterios.

Institucionalizar la perspectiva de género en la educación superior significa incluir el principio de la igualdad de género en las prácticas y estructuras que organizan la vida universitaria, de manera que este valor esté presente tanto en el contenido del conocimiento, como en su desarrollo como proceso. Es decir, institucionalizar un sistema de creencias que redunden en prácticas favorables a la igualdad implica un proceso de largo aliento que se sostiene mediante el cambio en la orientación de las políticas dentro de la institución universitaria y la adopción de prácticas organizativas explícitas que se asienten en mandatos institucionales permanentes.

\section{Aspectos centrales:}

- Voluntad político administrativa explícita, que se manifieste en mandatos institucionales y definición de acciones a favor de la igualdad de género en el nivel más alto de la jerarquía institucional. Es necesario el respaldo político e institucional que asiente los procesos de cambio y los haga sostenibles en el tiempo. La institucionalización de un nuevo enfoque como es el género debe ir más allá del compromiso e interés personal del grupo interesado y aunque las personas se muevan, la iniciativa continúa porque se consolida como parte de la institución.

- Creación de unidades de género en las universidades: Esta es una de las principales estrategias para institucionalizar la perspectiva de género, la que va acompañada de la elaboración de programas sustentados en diagnósticos sobre 
la población universitaria. Los diagnósticos, por su parte, nos permiten plantearnos cuál son las prácticas que en el ejercicio universitario requerimos transformar a la luz de los resultados de la investigación. Es preciso que las propuestas no queden inermes, sin una vinculación explícita con el compromiso de las autoridades ante los resultados y sus implicaciones a la vida universitaria. Aquí emerge el aspecto político que implica IPG dentro de instituciones de educación superior, en el entendido que la voluntad política es una de las variables centrales en el proceso. Unidades específicas junto con una estrategia de transversalidad deben considerarse como medidas que en su conjunto impulsen acciones a favor de las políticas de igualdad en las IES.

- Definir la IPG de manera específica para la vida universitaria debe incluir un diseño institucional donde las sanciones, pero también los incentivos hacia buenas prácticas en materia de actuación a favor de la igualdad de género estén presentes. Para ello es preciso conocer la vida interna de la organización educativa bajo un análisis organizacional con perspectiva de género y producir mecanismos de comunicación internos y externos que convoquen y sumen compromisos y voluntades de todos los sectores de la comunidad universitaria.

- Formación y capacitación en género para transferir y legitimar un conocimiento más allá de los y las especialistas en el tema. La reflexión sobre el tema de género con el personal de la organización en todos los niveles -académicos, estudiantes, administrativos- contribuye a la generación de un ambiente favorable a la temática y mayor involucramiento y compromiso.

Que las instituciones de educación superior se planteen el reto de mirarse críticamente sobre la actuación que tienen en relación a la reproducción de la discriminación de género, plantea un gran desafío. Es preciso que, además de proponer cambios que vinculen las políticas educativas y sus principios rectores con la igualdad de género, se institucionalicen los valores de la igualdad, respeto y no discriminación, en un sentido mucho más profundo que el que supone que tanto mujeres como varones tienen permitido ingresar a la universidad.

En síntesis, institucionalizar el enfoque de género dentro de las IES implica dar cuenta de cuáles son las inequidades existentes, y en qué ámbitos éstas se manifiestan, estableciendo medidas claras y continuas para revertirlas, pero por sobre todo, generar una conciencia de comunidad que comparte el valor de la igualdad como un principio rector del quehacer universitario.

La experiencia acumulada en los procesos de incorporación de la perspectiva de género en diversos ámbitos institucionales y organizacionales (Cerva 2013, 2017) me permite sostener que, junto con el establecimiento de procesos a favor de la equidad, emergen una serie de problemas y resistencias que es preciso contemplar: 
-La falta de convicción sobre la igualdad de género, de las y los dirigentes

-La política de simulación sobre la construcción de la igualdad de género.

-La falta de continuidad del presupuesto asignado para fomentar la igualdad de género.

-La falta de fortalecimiento a los centros y programas de género, más allá del compromiso del personal abocado a estas unidades

-El cambio de administraciones y la falta de sostenibilidad político administrativa de las estructuras y normas institucionales a favor de la igualdad de género.

- La retórica de lo políticamente correcto que favorece que se perpetúen y resignifiquen situaciones de discriminación y desigualdad.

Existe clara conciencia de parte de las participantes de las entrevistas de la necesidad de institucionalizar los procesos de incorporación de la perspectiva de género dentro de la Universidad, a partir de acciones concretas y concertadas que vayan más allá de políticas de difusión. En el discurso de las académicas, aun cuando la Universidad es representada como una institución de vanguardia y progresista dentro del marco nacional, sus prácticas institucionales no están ajenas a las relaciones de poder que se derivan de la configuración de género.

\section{Reflexiones finales}

Los procesos y prácticas que se dan dentro de las instituciones de educación superior reflejan una serie de estereotipos, creencias y expectativas sobre las relaciones de género que son transmitidas dentro de la organización. La distinción entre lo masculino y lo femenino refuerza el ejercicio de actividades concretas, pensamientos y creencias que se manifiestan en la interacción individual y colectiva, en el uso del lenguaje y en varios símbolos que refuerzan las estructuras genéricas. Más allá de los comportamientos individuales, existe un entorno social que interviene en la forma en que los sujetos aprenden y reproducen ciertos valores y actitudes en torno al género y deben ser analizados atendiendo a las dinámicas de la cultura y ambiente organizacional donde se producen.

Las universidades, como organizaciones, han probado ser ciegas al género en lo que respecta a su estructura y cultura organizacional. Hay evidencias empíricamente observables que indican que mujeres y hombres no sólo están en posiciones diferentes en la estructura piramidal de funciones y cargos, sino que además se ven afectados de manera diferencial por los estereotipos de género que en el ámbito universitario se reproducen. 
A ello debemos agregar las serias restricciones que enfrentan las unidades de género creadas específicamente para promover trasformaciones positivas al interior de las universidades. Las resistencias deben ser tomadas en serio, sobre todo considerado el papel que tienen las universidades en el entorno social.

Aspectos ligados al mérito, las promociones, las dinámicas de discriminación y las posibilidades de cambio son sumamente necesarias de investigar y promover

Con todo lo anterior, podemos sostener que existe un consenso en señalar que es preciso institucionalizar la perspectiva de género en la educación superior con el objetivo de incluir el principio de la igualdad de género en las prácticas y estructuras que organizan la vida universitaria, de manera que este valor esté presente tanto en el contenido del conocimiento, como en su desarrollo como proceso. Es decir, institucionalizar un sistema de creencias a favor del reconocimiento del valor de la igualdad y la diversidad requiere nombrar y reconocer las inequidades existentes, y en qué ámbitos éstas se manifiestan, estableciendo medidas claras y continuas para revertirlas, pero por sobre todo, generar una conciencia de comunidad que comparte el valor de la igualdad como un principio rector del quehacer universitario.

Reformas normativas, creación de mecanismos o unidades de género, generación de políticas institucionales de difusión de la noción de equidad, transformación de programas de estudio, entre otras medidas, han sido las estrategias seguidas para institucionalizar el género en las universidades.

\section{BIBLIOGRAFÍA}

Bellucci, Mabel (1992): "De los estudios de la mujer a los estudios de género: han recorrido un largo camino", en Fernández A. M. (compiladora.): Las mujeres en la imaginación colectiva, Buenos Aires, Paidós.

Blázquez, Norma, Fátima Flores y Maribel Ríos (coordinadoras) (2010): Investigación feminista. Epistemología, metodología y presentaciones sociales, México, $\mathrm{ClICH}$, CRIM, UNAM.

Buquet Corleto, A., Copper A, J., Rodríguez Loredo, H. (2010): Sistema de Indicadores para la Equidad de Género en la Educación Superior. INMUJERES/PUEGUNAM.

Buquet Corleto, A., (2011): "Transversalización de la perspectiva de género en la educación superior. Problemas conceptuales y prácticos", en Perfiles Educativos vol.33 p.221-225.

Buquet, Ana, et. al, (2013): Intrusas en la Universidad, México PUEG, UNAM. 
Centro de Investigación en Estudios de Género y Equidad (CIEGyE) (2012): Encuesta al personal administrativo sobre clima laboral y equidad de género, mayo, Toluca: CIEGyE/UAEM.

Cerva, Daniela, (2017): "Los espacios cuentan: prácticas y discursos en la administración pública sobre las políticas de igualdad de género", en Revista de Administración Pública (RAP) del Instituto Nacional de Administración Pública A.C, (en prensa).

Cerva, Daniela, (2016): “Masculinidades y construcción discursiva sobre las políticas de género en México", en Revista Cuestiones de género: de la igualdad y la diferencia. № 11, pp. 209-226 Universidad de León, España.

Cerva, Daniela (2013): “Procesos de institucionalización de la perspectiva de género en el Estado: Análisis desde la cultura organizacional”, en Juliana Ströbele-Gregor y DörteWallrad (editoras): Espacios de Género Alemania-Argentina, Friedrich Ebert Stiftung y Nueva Sociedad.

Cerva, Daniela (2011): Informe de Investigación: Resultados de los grupos focales realizados en la población académica y población administrativa de la Facultad de Psicología, Facultad de Derecho y Facultad de Ingeniería de la Universidad Autónoma de México. México PUEG UNAM (documento mimeografiado)

Cerva, Daniela (2009): “Cultura organizacional e institucionalización de las políticas de género en México: Notas para el debate" en Revista GénEros, Universidad de Colima, $N^{\circ} 6$, Epoca 2/Año 16, septiembre - febrero pp. 5570

Goldsmith Connelly, Mary, (1998): “Feminismo e investigación social: Nadando en aguas revueltas" en Eli Bartra (Comp): Debates en torno a una metodología feminista. México DF: UAM-X, CSH.

Guil Bozal, Ana, (2004): Techo de Cristal en la Universidad de Sevilla, España Universidad de Sevilla.

Kettle, Jane (1996): "Good practices, bad attitudes; and examination of the factoirs influencing women's academic careers", en Morley, Louise, y Vals Walsh (Eds.): Breaking boundaries: Woman and Higher education. Brisitol: Taylor y Francis.

Lagarde, Marcela, (1996): Género y feminismo. Desarrollo humano y democracia, España. Ed. horas y HORAS.

León, Magdalena, (2007): “Tensiones presentes en los estudios de género" en Luz Gabriela Arango y Yolanda Puyana (compiladora): Género, mujeres y saberes en 
América Latina. Entre el movimiento social, la academia y el Estado, Bogotá, Colombia, Universidad Nacional de Colombia. pp. 23-46.

Maffía, Diana (2000): "Las mujeres y la construcción de la ciencia”, en Palacios, María Julia y Cornejo, Raquel (ed): Latinoamérica. Fin de siglo: Utopías, Realidades, Proyectos, Salta, Universidad Nacional de Salta.

Maffía, Diana (2007): “Epistemología feminista: la subversión semiótica de las mujeres en la ciencia", en Revista Venezolana de Estudios de la Mujer № 28 "Filosofía Feminista".

Maffia, Diana, (2010): Género y Políticas Públicas en Ciencias y Tecnología, en Norma Blazquez, Fátima Flores y Maribel Ríos (coordinadoras): Investigación feminista. Epistemología, metodología y presentaciones sociales, México, CIICH, CRIM, UNAM.

Morley, Louise, (1999): "Techo de cristal o jaula de Hierro. Las mujeres en el medio académico del Reino Unido", en M. Belousteguigoitía, A. Mingo (coord): Géneros Prófugos, PUEG, UNAM, 1999. México.

Morley, Louise (2010): “Women's Careers in Higher Education: Theorising Gender Inequalities", en Training and Research Network, Women in European Universities. [On line] Disponible en: http://csn.uni-muenster.de/women-eu/

Palomar Vera, Cristina. (2011): La cultura institucional de género en la Universidad de Guadalajara. México, D.F.: ANUIES.

Rietti, Sara (1999): “Democratización del conocimiento. La perspectiva desde una política para la ciencia y el desarrollo educativo". [On line] Disponible en http://www.campus-oei.org/salactsi/sara3.htm

Rietti, Sara y Diana Maffía (2014): “Ciencia y Política, un vínculo necesario" en Perspectivas № 25, Isis Internacional, Santiago de Chile, abril-junio pp. 3-11.

Tubert, Silvia (ed) (2011): Del sexo al Género. Los equívocos de un concepto. España, Ediciones Cátedra, Universidad de Valencia.

Vélez, Graciela (2008): La construcción social del sujeto político femenino. Un enfoque identitario subjetivo. México: Porrúa/UAEM.

Vélez, Graciela y Vizcarra, Ivonne (2007): “Género y éxito científico en la Universidad Autónoma del Estado de México", en Revista de Estudios feministas vol. 15, 2007. Florianópolis, 15(3): 336 setembro-decembroBrasil. págs. 581-608. 
Revista Punto Género № 8. Diciembre de 2017

ISSN 0719-0417 / 20 - 38

Wenneras, Christine y Wold, Agnes, (1997): "Nepotism and sexism in peer-review", en Nature, Vol. 387/22, Macmillan Publishers Ltd.pp. 341-343.

I Reunión Nacional de Universidades Públicas: Caminos para la Equidad de Género en las Instituciones de Educación Superior, México. http://equidad.pueg.unam.mx/node/2 